\title{
Prognostic analysis of very early onset pancreatic cancer: a population based analysis
}

\author{
Dongjun Dai ${ }^{1}$, Yanmei Wang $^{1}$, Xinyang Hu ${ }^{1}$, Hongchuan Jin ${ }^{2}$, Xian Wang ${ }^{\text {Corresp. } 1}$ \\ ${ }^{1}$ Department of Medical Oncology, Sir Run Run Shaw Hospital, Medical School of Zhejiang University, Zhejiang University, Hangzhou, Zhejiang, China \\ Zhejiang, China \\ Corresponding Author: Xian Wang \\ Email address: wangx118@zju.edu.cn
}

Background : We aimed to use competing risk model to assess whether very early onset pancreatic cancer ( VEOPC ) (< 45 years) had a worse prognosis than older pancreatic cancer (PC) patients, and to build a competing risk nomogram for predicting the risk of death of VEOPC.

Methods: We selected pancreatic adenocarcinoma (PDAC) patients as our cohort from Surveillance, Epidemiology, and End Results (SEER) database. The impact of cancer specific death was estimated by competing risk analysis. Multivariate Fine-Gray regression for proportional hazards modeling of the subdistribution hazard (SH) model based nomogram was constructed, which was internally validated by discrimination and calibration with 1,000 bootstraps.

Results: Our cohort included 1,386 VEOPC patients and 53,940 older patients. We observed that in unresectablePDAC patients, VEOPC had better cancer specific survival (CSS) than each older group (45-59 years, 60-69 years, 70-79 years and > 79 years). There was no significant prognostic difference between VEOPC and each older group in resectablePDAC. Our competing nomogram showed well discrimination and calibration by internal validation.

Conclusion: For unresectable PDAC patients, VEOPC had better CSS than older patients. Our competing risk nomogram might be an easy-to-use tool for the specific death prediction of VEOPC patients with PDAC. 


\section{Prognostic analysis of very early onset pancreatic}

\section{2 cancer: a population based analysis}

3

Dongjun Dai ${ }^{1}$, Yanmei Wang ${ }^{1}$, Xinyang Hu${ }^{1}$, Hongchuan $\mathrm{Jin}^{2}$, Xian Wang ${ }^{1, *}$

1 Department of Medical Oncology, Sir Run Run Shaw Hospital, Medical School of Zhejiang University, Zhejiang University, Hangzhou, China;

2 Laboratory of Cancer Biology, Key Lab of Biotherapy, Sir Run Run Shaw Hospital, Medical School of Zhejiang University, Zhejiang University, Hangzhou, China

Corresponding Author:

Xian Wang ${ }^{1}$

Department of Medical Oncology, Sir Run Run Shaw Hospital, Medical School of Zhejiang University, Zhejiang University, 3 East Qingchun Road, Hangzhou, China Email address: wangx118@zju.edu.cn

\section{Abstract}

Background: We aimed to use competing risk model to assess whether very early onset pancreatic cancer (VEOPC) $(<45$ years) had a worse prognosis than older pancreatic cancer (PC) patients, and to build a competing risk nomogram for predicting the risk of death of VEOPC.

Methods: We selected pancreatic adenocarcinoma (PDAC) patients as our cohort from Surveillance, Epidemiology, and End Results (SEER) database. The impact of cancer specific death was estimated by competing risk analysis. Multivariate Fine-Gray regression for proportional hazards modeling of the subdistribution hazard $(\mathrm{SH})$ model based nomogram was constructed, which was internally validated by discrimination and calibration with 1,000 bootstraps.

Results: Our cohort included 1,386 VEOPC patients and 53,940 older patients. We observed that in unresectable PDAC patients, VEOPC had better cancer specific survival (CSS) than each older group (45-59 years, 60-69 years, 70-79 years and $>79$ years). There was no significant prognostic difference between VEOPC and each older group in resectable PDAC. Our competing nomogram showed well discrimination and calibration by internal validation. 
Conclusion: For unresectable PDAC patients, VEOPC had better CSS than older patients. Our competing risk nomogram might be an easy-to-use tool for the specific death prediction of VEOPC patients with PDAC.

List of abbreviations: $P C$, pancreatic cancer; VEOPC, very early onset pancreatic cancer; BMI, body mass index; OS, overall survival; PDAC; pancreatic adenocarcinoma; SEER, Surveillance, Epidemiology, and End Results; NOS, not otherwise specified; LNR, lymph nodes ratio; AJCC, American Joint Committee on Cancer; CID, cumulative incidences of death; CIF, cumulative incidences function; CSS, cancer specific survival; $\mathrm{HR}$, hazard ratio; $95 \% \mathrm{Cl}, 95 \%$ confidence index; $\mathrm{AUC}$, the area under the curve of receiver operating characteristic curve.

\section{Introduction}

Pancreatic cancer (PC) patients have a 5 years survival lower than $10 \%$ (Siegel et al. 2018). According to American cancer society, the average age of PC patients at diagnosis is 70 years. Less than $3 \%$ of PC patients are under the age of 45 years at diagnosis (Surveillance 2019a), which was defined as very early onset PC (VEOPC)(McWilliams et al. 2016). Duo to its rareness, few data were reported regarding the clinicopathological features and treatment response of VEOPC patients(Grosfeld et al. 1990; Jaksic et al. 1992; Lack et al. 1983; McWilliams et al. 2016; Shorter et al. 2002).

The identification of prognostic factors of PC might improve the prediction of the survival and the selection of therapy for PC patients. The well-known PC prognostic factors comprised resection margins, lymph nodes status and lymph node ratio, perineural and blood vessel invasion, tumor localization and body mass index (BMI) and treatment such as chemotherapy(Bilici 2014; Lin et al. 2016). However, whether age is a prognostic factor of PC is currently controversial. Some studies found "younger" PC patients (with different definition of age $<45$ years, age $<50$ years, age $<60$ years and age $<70$ years) had better overall survival (OS) than the older PC patients(Baxter et al. 2007; He et al. 2013; Saad et al. 2018; Sugiura et al. 2017). While the other studies found younger age (with different definition of age $<65$ years and age $<70$ years)(Barbas et al. 2012; Miyazaki et al. 2016; van der Geest et al. 2016) was not associated with the survival of PC patients. To be noted, these studies had limited number of VEOPC patients or did not treat the VEOPC as a group. 
68

Nomogram is a valuable tool to use different clinical variables to determine a statistical prognostic model that generates a probability of clinical outcomes for a single patient(Balachandran et al. 2015). Nomogram have been applied in various types of cancers(Fakhry et al. 2017; Huang et al. 2016; Liang et al. 2015; Rose et al. 2015). Nowadays, nomograms are commonly used to estimate prognosis in oncology and medicine, which fit the trend of personalized medicine(Balachandran et al. 2015). It is worthy to construct a nomogram for predicting the prognosis of VEOPC patients.

Pancreatic adenocarcinoma (PDAC) is the main type of PC, which has a worse prognosis than other histology types. The current study selected PDAC patients as our cohort. To be noted, the competing event of death is widely existing in the prognostic analysis of cancer. The failure to account for such competing events, such as KaplanMeier method and standard Cox proportional hazards regression analysis, might result in an overestimate of the cumulative incidences(Satagopan et al. 2004). The competing risk model considers both the disease-specific death and other causes of death, which was fit to the analysis in the presence of competing risk events (Kim 2007). The competing risk analysis was often missed in previous studies when included with very aged PC patients. The aim of our study was to use competing risk model to estimate the prognostic value of age in PDAC patients and construct competing nomogram for cancer-specific death prediction of VEOPC patients by using the data from Surveillance, Epidemiology, and End Results (SEER) database of the National Cancer Institute of United States, which currently collects and publishes cancer incidence and survival data from population-based cancer registries covering approximately 34.6 percent of the United States population(Surveillance 2019b).

\section{Methods}

\section{Cohort selection}

The cohort was selected by SEER*Stat 8.3 .5 software (SEER ID: daid) with the following criteria: (1) it should be a primary PDAC patients (ICD-O-3 histology codes 8140 and 8500 ) registered equal or after year of 2004; (2) it should be a patient who has a survival time over than 0 month with records of survival status; (3) it should have at least one year follow up time to capture enough events to ensure a meaningful analysis. We also excluded few patients with unclear information of race, surgery, number of regional lymph nodes examined, radiotherapy and marital status. Finally, we excluded 1 patient with cancer in the islets of langerhans. The detailed data selection process was shown in Supplemental Figure 1. 


\section{Study variables and endpoints}

We included 14 variables into our analysis, which were listed as the follows: age at diagnosis, sex (Females and males), race (Caucasian, African American, American Indian/Alaska Native and Asian or Pacific Islander), tumor location (Head of pancreas, body of pancreas, tail of pancreas, pancreatic duct, other specified parts of pancreas, overlapping lesion of pancreas and Pancreas, NOS (Not otherwise specified)), surgery experience (No surgery, local or partial pancreatectomy, local or partial pancreatectomy and duodenectomy, total pancreatectomy with or without gastrectomy or duodenectomy, pancreatectomy NOS or surgery NOS), tumor size, LNR (Lymph nodes ratio, which is defined as the ratio of the number of positive lymph nodes to the total number of lymph nodes examined), 6th American Joint Committee on Cancer (AJCC) tumor TNM stage, grade, radiotherapy and chemotherapy experience and marital status. The value of age at diagnosis, tumor size and LNR were classified into small categorical variables to fit the linear assumption. The widowed or single (Never married or having a domestic partner) or divorced or separated patients was defined as "Single". The median observed survival time was referred to median follow-up time.

\section{Statistical analyses}

The difference of each variable between VEOPC and other PC patients was evaluated by Chi-Squared tests. Cumulative incidences of death (CID) was estimated for deaths caused by cancer or other reasons by using the cumulative incidences function (CIF) analysis. Multivariate SH model was used to assess the cancer specific survival (CSS). All the variables were included in the multivariate analysis. Hazard ratio (HR) and 95\%confidence index $(95 \% \mathrm{Cl})$ were calculated.

Multivariate $\mathrm{SH}$ model-based nomogram was constructed by multivariate logistic regression model to predict the 6 months, 12 months, 18 months and 24 months cancer specific death of VEOPC patients. The internal validation of nomogram was performed by discrimination and calibration with 1,000 time bootstraps(Balachandran et al. 2015). The discrimination was estimated by the area under the curve of receiver operating characteristic curve (AUC, which was also referred as C-statistics). The AUC ranges from 0.5-1.0, with 0.5 indicates the outcomes is totally random and 1.0 indicates the perfect discrimination. The calibration was assessed by calibration curves, which shows how close the nomogram estimated risk was to the observed risk. All the statistical analyses were performed by "R" version 3.6.0. The CIF test and multivariate $\mathrm{SH}$ analysis were performed by "R" package "cmprsk". The competing risk nomogram was constructed by 
"R" package "mstate" and plotted by "R" package "regplot". The calibration curve was drawn by "R" package "riskRegression". The AUC was calculated by "R" package "riskRegression" and plotted by "R" package "ggplot2". A two tailed p-value less than 0.05 was considered statistically significant.

\section{Results}

\section{Cohort selection}

We included the patients diagnosed equal to or before 2015 to ensure an enough follow-up time. There were 1,386 VEOPC patients and 53,940 older PDAC patients (>= 45 years). The median survival time of VEOPC and older patients were 9 and 7 months, respectively. Significant differences were found between VEOPC and other PC patients. The VEOPC patients had more males, fewer Caucasians, more surgery rates, fewer LNR, higher TNM stage, higher grade, more experience of chemotherapy and radiotherapy and fewer of married status ( $p<0.001$, Table 1$)$ than the older patients. We also provided a table with characteristics for all patients (Supplemental Table 1). We divided the older patients into 4 groups with different age ranges (45-59 years, 60-69 years, 70-79 years and $>79$ years) for further analysis.

\section{Prognostic analysis of age in PDAC patients}

The CIF plot showed VEOPC patients had a decreased risk of cancer death than older patients $(p<0.001$, Figure 1a). Further subgroup analyses by surgery showed that VEOPC patients had better prognosis than the older patients whether they performed surgery or not $(p<0.001$, Figure $1 b-c)$. Moreover, multivariate $\mathrm{SH}$ model with all the included variables showed that in unresectable PDAC patients, the VEOPC had better CSS than older patients. While in the resected PDAC patients, no significant difference was found between VEOPC and older patients (Table 2).

\section{Independent prognostic factors of CSS of VEOPC}

As shown in Table 3, multivariate $\mathrm{SH}$ model was used to find independent prognostic factors for the CSS of VEOPC patients. We found the female, race of Asian or Pacific Islander, PC located in pancreatic duct, no surgery, high LNR, high M stage, high grade, lack of chemotherapy, and single status were risk factors of the VEOPC patients $(P<$ $0.05)$.

\section{Nomogram development and validation}


174

175

176

177

178

179

180

181

182

183

184

185

186

187

188

189

190

191

192

193

194

195

196

197

198

199

200

201

202

203

204

205

206

207

208

The nomogram was constructed by multivariate $\mathrm{SH}$ model. A weighted total score calculated from each variable was used to estimate the 6 months, 12 months, 18 months and 24 months cancer specific death of VEOPC patients (Figure 2). The sex, race, location, surgery strategy, tumor size, LNR, TNM stage, grade, chemotherapy and marital status were found to be significantly important for the prognosis of VEOPC patients (Variables with asterisk in Figure 2). The calibration plots showed well correlation between observed CSS and nomogram predicted CSS (Figure 3a-d).Time dependent AUC plot suggested well discrimination of our nomogram (AUC $>=0.736$, Figure $3 e$ ). Furthermore, we equally stratified the whole VEOPC into three groups based on their level of competing nomogram scores and performed a CIF analysis. The CIF plot (Figure 4) showed VEOPC with higher scores of our competing nomogram was at high risk of cancer related death. It indicated that our competing nomogram was useful for risk stratification of the prognosis of VEOPC patients. The detailed competing nomogram scores for all variables were listed in Supplemental Table 2.

\section{Discussion}

No previous competing analysis was performed to analyze the prognostic difference between VEOPC and older PC patients. The current study used competing risk model to estimate the prognostic difference between VEOPC and older patients by using the data from SEER. We found that among the unresectable PDAC patients, the VEOPC had better CSS than older patients. And among resected PDAC patients, there was no significant prognostic difference between VEOPC and older patients. We also built the first competing nomogram for primary VEOPC patients with histology type of PDAC.

Age was observed as an independent prognostic factor for many cancers. Previous studies identified that younger patients had worse prognosis than older patients in colorectal cancer(van Eeghen et al. 2015) and breast cancer(Chen et al. 2016) while younger patients had better prognosis than older patients in lung cancer(Arnold et al. 2016), liver cancer(Zhang \& Sun 2015) and prostate cancer (Pettersson et al. 2018). A study found that the $<45$ years PDAC patients had better survival than $>70$ years PDAC patients (He et al. 2013). Another study found $<50$ years PDAC patients had no significant difference on prognosis when compared with their whole cohort(Tingstedt et al. 2011). To be noted, these studies only included with limited samples. A SEER based study found the risk of cancer-specific death decreases with age in resectable PDAC patients(He et al. 2018). However, we found the age was not an independent prognostic factor for resectable PDAC patients. This difference might be caused by the difference of inclusion 
criteria that our study included more variables in the multivariate $\mathrm{SH}$ model. We provided a detailed inclusion workflow in the Supplemental Figure 1. We found the VEOPC patients, despite with a higher TNM stage, had a better CSS than the older patients in unresectable patients (Supplemental Table 3). It might be explained by the well accepted fact that younger patients had more tolerance to chemotherapy. Indeed, in the unresectable group, the VEOPC patients had a $17.5 \%$ higher rate to have chemotherapy than the older patients.

Males had higher rate of PC incidence in United States(Yadav \& Lowenfels 2013). We observed the males had $15 \%$ higher rate than the females in VEOPC. Previous study found males had worse prognosis than females in patients under 60 years but not in VEOPC patients(McWilliams et al. 2016). It might be caused by the sample size. Our study had much higher amount of VEOPC patients than this study and we found the males had worse CSS than females.

Marriage status was linked to the improvements in cardiovascular, endocrine, immune function, and cancer prognosis(Aizer et al. 2013; Gallo et al. 2003; Herberman \& Ortaldo 1981). Previous study observed that unmarried patients were at significantly higher risk of presentation with metastatic cancer, undertreatment, and death resulting from their cancer(Aizer et al. 2013). We found the VEOPC patients with married status had better CSS than patients with single status, which provided new hint to the association between support care and cancer prognosis.

Our SH model based nomogram was well validated by discrimination and calibration. The variables included in our nomogram were easy to be obtained, allowing a feasible translation into the future clinical use. To be noted, we included both the significant variables and the insignificant variables into our nomogram, since if a nomogram only includes statistically significant variables, it might tend to exert an inappropriately large influence, leading to falsely narrowed confidence intervals, which would make this nomogram appear more accurate than it is(Nicholas J Vogelzang 2005). Patients with higher competing nomogram scores of our study were found to have higher CID, indicating that our nomogram was useful for risk stratification of the prognosis of VEOPC patients.

Our study had some limitations. First, the detailed strategy of chemotherapy or radiotherapy was missed in current study as well as other therapies such as immunotherapy. Second, although the current nomogram was well validated by internal validation, there was a lack of an external validation by cohort other than SEER. Third, it should be noted that only factors available in the SEER registry were examined. The results from this study might only fit the population from United States. Fourth, our study 
245

246

247

248

249

250

251

252

253

254

255

256

257

258

259

260

261

262

263

264

265

266

267

268

269

270

271

272

273

274

275

276

277

278

279

280

281

282

has no exploration of molecular mechanism, which was of interest but cannot be conducted at this time with data currently available.

\section{Conclusions}

The current study showed that for unresectable PDAC, VEOPC had better CSS than older patients. For the VEOPC patients, we built a SH model based nomogram to estimate their CSS. This nomogram was shown to have good discrimination and calibration.

\section{References}

Aizer AA, Chen MH, McCarthy EP, Mendu ML, Koo S, Wilhite TJ, Graham PL, Choueiri TK, Hoffman KE, Martin NE, Hu JC, and Nguyen PL. 2013. Marital status and survival in patients with cancer. J Clin Oncol 31:3869-3876. 10.1200/JCO.2013.49.6489

Arnold BN, Thomas DC, Rosen JE, Salazar MC, Blasberg JD, Boffa DJ, Detterbeck FC, and Kim AW. 2016. Lung Cancer in the Very Young: Treatment and Survival in the National Cancer Data Base. J Thorac Oncol 11:1121-1131. 10.1016/j.jtho.2016.03.023

Balachandran VP, Gonen M, Smith JJ, and DeMatteo RP. 2015. Nomograms in oncology: more than meets the eye. Lancet Oncol 16:e173-180. 10.1016/S1470-2045(14)71116-7

Barbas AS, Turley RS, Ceppa EP, Reddy SK, Blazer DG, 3rd, Clary BM, Pappas TN, Tyler DS, White RR, and Lagoo SA. 2012. Comparison of outcomes and the use of multimodality therapy in young and elderly people undergoing surgical resection of pancreatic cancer. J Am Geriatr Soc 60:344-350. 10.1111/j.15325415.2011.03785.x

Baxter NN, Whitson BA, and Tuttle TM. 2007. Trends in the treatment and outcome of pancreatic cancer in the United States. Ann Surg Oncol 14:1320-1326. 10.1245/s10434-006-9249-8

Bilici A. 2014. Prognostic factors related with survival in patients with pancreatic adenocarcinoma. World $J$ Gastroenterol 20:10802-10812. 10.3748/wjg.v20.i31.10802

Chen HL, Zhou MQ, Tian W, Meng KX, and He HF. 2016. Effect of Age on Breast Cancer Patient Prognoses: A Population-Based Study Using the SEER 18 Database. PLoS One 11:e0165409. 10.1371/journal.pone.0165409

Fakhry C, Zhang Q, Nguyen-Tan PF, Rosenthal DI, Weber RS, Lambert L, Trotti AM, 3rd, Barrett WL, Thorstad WL, Jones CU, Yom SS, Wong SJ, Ridge JA, Rao SSD, Bonner JA, Vigneault E, Raben D, Kudrimoti MR, Harris J, Le QT, and Gillison ML. 2017. Development and Validation of Nomograms Predictive of Overall and Progression-Free Survival in Patients With Oropharyngeal Cancer. J Clin Oncol 35:4057-4065. 10.1200/JCO.2016.72.0748

Gallo LC, Troxel WM, Matthews KA, and Kuller LH. 2003. Marital status and quality in middle-aged women: Associations with levels and trajectories of cardiovascular risk factors. Health Psychol 22:453-463. 10.1037/0278-6133.22.5.453

Grosfeld JL, Vane DW, Rescorla FJ, McGuire W, and West KW. 1990. Pancreatic tumors in childhood: analysis of 13 cases. J Pediatr Surg 25:1057-1062. 
283

284

285

286

287

288

289

290

291

292

293

294

295

296

297

298

299

300

301

302

303

304

305

306

307

308

309

310

311

312

313

314

315

316

317

318

319

320

321

322

323

He C, Zhang Y, Cai Z, Lin X, and Li S. 2018. Overall survival and cancer-specific survival in patients with surgically resected pancreatic head adenocarcinoma: A competing risk nomogram analysis. J Cancer 9:3156-3167. $10.7150 /$ jca. 25494

He J, Edil BH, Cameron JL, Schulick RD, Hruban RH, Herman JM, Zheng L, lacobuzio-Donahue C, Ahuja N, Pawlik TM, and Wolfgang CL. 2013. Young patients undergoing resection of pancreatic cancer fare better than their older counterparts. J Gastrointest Surg 17:339-344. 10.1007/s11605-012-2066-4

Herberman RB, and Ortaldo JR. 1981. Natural killer cells: their roles in defenses against disease. Science 214:24-30. 10.1126/science. 7025208

Huang YQ, Liang CH, He L, Tian J, Liang CS, Chen X, Ma ZL, and Liu ZY. 2016. Development and Validation of a Radiomics Nomogram for Preoperative Prediction of Lymph Node Metastasis in Colorectal Cancer. J Clin Oncol 34:2157-2164. 10.1200/JCO.2015.65.9128

Jaksic T, Yaman M, Thorner P, Wesson DK, Filler RM, and Shandling B. 1992. A 20-year review of pediatric pancreatic tumors. J Pediatr Surg 27:1315-1317.

Kim HT. 2007. Cumulative incidence in competing risks data and competing risks regression analysis. Clin Cancer Res 13:559-565. 10.1158/1078-0432.CCR-06-1210

Lack EE, Cassady JR, Levey R, and Vawter GF. 1983. Tumors of the exocrine pancreas in children and adolescents. A clinical and pathologic study of eight cases. Am J Surg Pathol 7:319-327.

Liang W, Zhang L, Jiang G, Wang Q, Liu L, Liu D, Wang Z, Zhu Z, Deng Q, Xiong X, Shao W, Shi X, and He J. 2015. Development and validation of a nomogram for predicting survival in patients with resected non-small-cell lung cancer. J Clin Oncol 33:861-869. 10.1200/JCO.2014.56.6661

Lin JY, Zhang XM, Kou JT, Fa H, Zhang XX, Dai Y, and He Q. 2016. Analysis of prognostic factors for pancreatic head cancer according to para-aortic lymph node. Cancer Med 5:2701-2707. 10.1002/cam4.853

McWilliams RR, Maisonneuve P, Bamlet WR, Petersen GM, Li D, Risch HA, Yu H, Fontham ET, Luckett B, Bosetti C, Negri E, La Vecchia C, Talamini R, Bueno de Mesquita HB, Bracci P, Gallinger S, Neale RE, and Lowenfels AB. 2016. Risk Factors for Early-Onset and Very-Early-Onset Pancreatic Adenocarcinoma: A Pancreatic Cancer Case-Control Consortium (PanC4) Analysis. Pancreas 45:311-316. 10.1097/MPA.0000000000000392

Miyazaki Y, Kokudo T, Amikura K, Kageyama Y, Takahashi A, Ohkohchi N, and Sakamoto H. 2016. Age does not affect complications and overall survival rate after pancreaticoduodenectomy: Single-center experience and systematic review of literature. Biosci Trends 10:300-306. 10.5582/bst.2016.01093

Nicholas J Vogelzang PTS, William U Shipley, Frans MJ Debruyne, W. Marston Linehan. 2005. Comprehensive Textbook of Genitourinary Oncology 3rd Edition: LWW; Third edition (September 15, 2005).

Pettersson A, Robinson D, Garmo H, Holmberg L, and Stattin P. 2018. Age at diagnosis and prostate cancer treatment and prognosis: a population-based cohort study. Ann Oncol 29:377-385. 10.1093/annonc/mdx742

Rose PG, Java J, Whitney CW, Stehman FB, Lanciano R, Thomas GM, and DiSilvestro PA. 2015. Nomograms Predicting Progression-Free Survival, Overall Survival, and Pelvic Recurrence in Locally Advanced Cervical Cancer Developed From an Analysis of Identifiable Prognostic Factors in Patients From NRG Oncology/Gynecologic Oncology Group Randomized Trials of Chemoradiotherapy. J Clin Oncol 33:2136-2142. 10.1200/JCO.2014.57.7122

Saad AM, Turk T, Al-Husseini MJ, and Abdel-Rahman O. 2018. Trends in pancreatic adenocarcinoma incidence and mortality in the United States in the last four decades; a SEER-based study. BMC Cancer 18:688. $10.1186 /$ s12885-018-4610-4

Peer) reviewing PDF | (2019:06:38653:1:1:REVIEW 5 Dec 2019) 
Satagopan JM, Ben-Porat L, Berwick M, Robson M, Kutler D, and Auerbach AD. 2004. A note on competing risks in survival data analysis. Br J Cancer 91:1229-1235. 10.1038/sj.bjc.6602102

Shorter NA, Glick RD, Klimstra DS, Brennan MF, and Laquaglia MP. 2002. Malignant pancreatic tumors in childhood and adolescence: The Memorial Sloan-Kettering experience, 1967 to present. J Pediatr Surg 37:887-892.

Siegel RL, Miller KD, and Jemal A. 2018. Cancer statistics, 2018. CA Cancer J Clin 68:7-30. 10.3322/caac.21442

Sugiura T, Okamura Y, Ito T, Yamamoto Y, Ashida R, and Uesaka K. 2017. Impact of Patient Age on the Postoperative Survival in Pancreatic Head Cancer. Ann Surg Oncol 24:3220-3228. 10.1245/s10434-017-5994-0

Surveillance E, and End Results Program. 2019a. Cancer Stat Facts: Pancreatic Cancer. Available at https://seer.cancer.gov/statfacts/html/pancreas.html.

Surveillance E, and End Results Program. 2019b. Overview of the SEER Program. Available at https://seer.cancer.gov/about/overview.html (accessed November 28th 2019).

Tingstedt B, Weitkamper C, and Andersson R. 2011. Early onset pancreatic cancer: a controlled trial. Ann Gastroenterol 24:206-212.

van der Geest LG, Besselink MG, van Gestel YR, Busch OR, de Hingh IH, de Jong KP, Molenaar IQ, and Lemmens VE. 2016. Pancreatic cancer surgery in elderly patients: Balancing between short-term harm and long-term benefit. A population-based study in the Netherlands. Acta Oncol 55:278-285. 10.3109/0284186X.2015.1105381

van Eeghen EE, Bakker SD, van Bochove A, and Loffeld RJ. 2015. Impact of age and comorbidity on survival in colorectal cancer. J Gastrointest Oncol 6:605-612. 10.3978/j.issn.2078-6891.2015.070

Yadav D, and Lowenfels AB. 2013. The epidemiology of pancreatitis and pancreatic cancer. Gastroenterology 144:1252-1261. 10.1053/j.gastro.2013.01.068

Zhang W, and Sun B. 2015. Impact of age on the survival of patients with liver cancer: an analysis of 27,255 patients in the SEER database. Oncotarget 6:633-641. 10.18632/oncotarget.2719

\section{Figure legends}

Figure 1. CIF plots of the association between age and the prognosis of PDAC patients Legends: a-c, The CIF plots of the association between age and the prognosis of all cohort (a), patients with surgery (b) or without surgery (c).

Figure 2. Nomogram for predicting 6 months, 12 months, 18 months and 24 months cancer specific death of VEOPC patients

Legends: The nomogram is used by summing the points identified on the top scale for each independent variable and drawing a vertical line from the total points scale to the 6 months, 12 months, 18 months and 24 months cancer specific death to obtain the probability of survival. The total points projected to the bottom scale indicate the probability of the 6 months, 12 months, 18 months and 24 months cancer specific death. Sex: 1 = Male, 2 = Female; Race: 1 = Caucasian, 2 = African American, $\mathrm{N}=$ Other race; 
362 Location: 250 = Head of pancreas, 251 = Body of pancreas, $252=$ Tail of pancreas, 253

$363=$ Pancreatic duct, $257=$ Other specified parts of pancreas, $258=$ Overlapping lesion of 364 pancreas, $259=$ Pancreas, NOS; Surgery, $0=$ No surgery, $1=$ Local or partial pancreatectomy, 2 = Local or partial pancreatectomy and duodenectomy, $3=$ Total pancreatectomy with or without gastrectomy or duodenectomy, $4=$ Pancreatectomy NOS or surgery NOS; Tumor size: $2=<=2 \mathrm{~cm}, 4=2 \mathrm{~cm}$ to $4 \mathrm{~cm}, 6=4 \mathrm{~cm}$ to $6 \mathrm{~cm}, 8=>6 \mathrm{~cm}, 9$ = Unknown; LNR: $0=0,1=<=0.2,2=0.2-0.4,3=>0.4,4=$ No nodes were examined; T stage: $1=\mathrm{T} 1-\mathrm{T} 2,2=\mathrm{T} 3-\mathrm{T} 4,9=$ Unknown; $\mathrm{N}$ stage: $0=\mathrm{NO}, 1=\mathrm{N} 1,9=$ Unknown; $\mathrm{M}$ stage: $0=\mathrm{M}$, 1 = M1, 9 = Unknown; Grade: 1 = I-II, $2=\mathrm{III}-\mathrm{IV}, 9=$ Unknown; Chemotherapy: $0=$ none/unknown and $1=$ yes; Radiotherapy: $0=$ none/unknown or refused, 1 = beam radiation or combination of beam with implants or isotopes or radiation with method or source not specified or radioactive implants or radioisotopes and $\mathrm{N}=$ Recommended, unknown if administered; Marital status: $0=$ married; $1=$ widowed or single (never married or having a domestic partner) or divorced or separated. The asterisk means significant.

Figure 3. Calibration curves and time dependent AUC plot for internal validation of $\mathrm{SH}$ based nomograms

Legends: a-d, the calibration plots for 6 months (a), 12 months (b), 18 months (c) and 24 months (d) cancer specific death prediction of VEOPC patients; The x-axis shows the nomogram predicted probability while the $y$-axis is the actual survival estimated by the $\mathrm{SH}$ method, the black thick line overlaps the grey line indicating near perfect calibration; e, time dependent AUC plots of SH-based nomograms.

Figure 4. CIF plot based on the competing nomogram scores for the VEOPC patients Legends: the whole cohort of VEOPC patients were divided into three equally distributed groups. The groups were named "High", "Intermediate" and "Low" according to the levels of patients' competing nomogram scores. 


\section{Table $\mathbf{1}$ (on next page)}

The demographic and treatment data of VEOPC and older PC ( $>=45$ years) patients

All variables showed significant differences between VEOPC and older PC patients $(p<$ $0.001)$. 
1 Table 1. The demographic and treatment data of VEOPC and older PC ( $>=45$ years)

\section{patients}

VEOPC Older PC

Characteristics

$(\mathrm{N}=1386)$

No. of patients \%

\section{Sex}

Male

Female

796

590

$57.4 \%$

27337

$50.7 \%$

Race

Caucasian

African American

American Indian/Alaska Native

Asian or Pacific Islander

\section{Location}

Head of pancreas

Body of pancreas

Tail of pancreas

Pancreatic duct

Other specified parts of pancreas

Overlapping lesion of pancreas

Pancreas, NOS

\section{Surgery}

No surgery

Local or partial

pancreatectomy

Local or partial

pancreatectomy and

duodenectomy

Total pancreatectomy with or without gastrectomy or

duodenectomy

Pancreatectomy NOS or

surgery NOS

\section{Tumor size (cm)}

$<=2$

2 to 4

4 to 6

$>6$

Unknown
1026

207

13

140

738

154

182

12

18

113

169

1040

43

242

50

11

144

524

333

104

281
$42.6 \%$

26595

$74.0 \% \quad 43089$

$14.9 \% \quad 6522$

$0.9 \% \quad 287$

$10.1 \% \quad 4034$

$53.2 \%$

$11.1 \%$

29410

6921

$13.1 \%$

6458

$0.9 \% \quad 319$

$1.3 \% \quad 805$

4049

$8.2 \%$

$12.2 \%$

5970

$54.5 \%$

$12.8 \%$

$12.0 \%$

$0.6 \%$

$1.5 \%$

$7.5 \%$

$11.1 \%$

$77.9 \%$

$75.0 \% \quad 41989$

$3.1 \% \quad 1517$

$2.8 \%$

$17.5 \% \quad 8175$

$15.2 \%$

1978

$3.7 \%$

$3.6 \%$

$0.8 \%$

273

$0.5 \%$

$10.4 \% \quad 4883$

$9.1 \%$

$37.8 \%$

23278

$43.2 \%$

$24.0 \% \quad 12388$

$23.0 \%$

$7.5 \%$

3840

$7.1 \%$

$20.3 \% \quad 9543$ 


\begin{tabular}{|c|c|c|c|c|}
\hline \multicolumn{5}{|l|}{ LNR } \\
\hline 0 & 137 & $9.9 \%$ & 4542 & $8.4 \%$ \\
\hline$<=0.2$ & 126 & $9.1 \%$ & 3983 & $7.4 \%$ \\
\hline $0.2-0.4$ & 57 & $4.1 \%$ & 2180 & $4.0 \%$ \\
\hline $0.4-1$ & 55 & $4.0 \%$ & 2116 & $3.9 \%$ \\
\hline No nodes were examined & 1011 & $72.9 \%$ & 41111 & $76.2 \%$ \\
\hline \multicolumn{5}{|l|}{ T stage } \\
\hline $\mathrm{T} 1-\mathrm{T} 2$ & 275 & $19.8 \%$ & 11774 & $21.8 \%$ \\
\hline T3-T4 & 865 & $62.4 \%$ & 33301 & $61.7 \%$ \\
\hline Unknown & 246 & $17.7 \%$ & 8857 & $16.4 \%$ \\
\hline \multicolumn{5}{|l|}{ N stage } \\
\hline NO & 603 & $43.5 \%$ & 26845 & $49.8 \%$ \\
\hline $\mathrm{N} 1$ & 584 & $42.1 \%$ & 19325 & $35.8 \%$ \\
\hline Unknown & 199 & $14.4 \%$ & 7762 & $14.4 \%$ \\
\hline \multicolumn{5}{|l|}{ M stage } \\
\hline M0 & 582 & $42.0 \%$ & 26570 & $49.3 \%$ \\
\hline M1 & 786 & $56.7 \%$ & 25678 & $47.6 \%$ \\
\hline Unknown & 18 & $1.3 \%$ & 1684 & $3.1 \%$ \\
\hline \multicolumn{5}{|l|}{ Grade } \\
\hline$|-| \mid$ & 326 & $23.5 \%$ & 11939 & $22.1 \%$ \\
\hline III-IV & 256 & $18.5 \%$ & 8755 & $16.2 \%$ \\
\hline Unknown & 804 & $58.0 \%$ & 33238 & $61.6 \%$ \\
\hline \multicolumn{5}{|l|}{ Chemotherapy } \\
\hline No & 312 & $22.5 \%$ & 20586 & $38.2 \%$ \\
\hline Yes & 1074 & $77.5 \%$ & 33346 & $61.8 \%$ \\
\hline \multicolumn{5}{|l|}{ Radiotherapy } \\
\hline No & 1021 & $73.7 \%$ & 42283 & $78.4 \%$ \\
\hline Yes & 365 & $26.3 \%$ & 11649 & $21.6 \%$ \\
\hline \multicolumn{5}{|l|}{ Marital status } \\
\hline Married & 768 & $55.4 \%$ & 32013 & $59.4 \%$ \\
\hline Single & 618 & $44.6 \%$ & 21919 & $40.6 \%$ \\
\hline
\end{tabular}

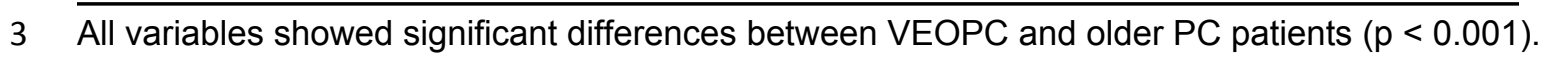




\section{Table 2 (on next page)}

Multivariate $\mathrm{SH}$ analysis of CSS for the association between age and the prognosis of PDAC patients

We included all variables in the $\mathrm{SH}$ multivariate analysis. HR, Hazard ratio; $95 \% \mathrm{Cl}$, $95 \%$ confidence index; Significant results $(p<0.05)$ were bolded. 
1 Table 2. Multivariate SH analysis of CSS for the association between age and the prognosis of PDAC patients

\begin{tabular}{|c|c|c|c|c|c|c|}
\hline \multirow{2}{*}{ Age (Years) } & \multicolumn{2}{|c|}{ For the whole cohort } & \multicolumn{2}{|c|}{ For patients with surgery } & \multicolumn{2}{|c|}{ For patients without surgery } \\
\hline & $\operatorname{HR}(95 \% \mathrm{Cl})$ & $p$ value & $\mathrm{HR}(95 \% \mathrm{Cl})$ & $\mathrm{p}$ value & $\operatorname{HR}(95 \% \mathrm{Cl})$ & $p$ value \\
\hline$<45$ & Reference & & Reference & & Reference & \\
\hline $45-59$ & $1.08(1.02-1$ & 0.010 & $0.98(0.86-1$ & $7.50 \mathrm{E}-01$ & $1.10(1.03-1.16)$ & 0.003 \\
\hline $60-69$ & $1.10(1.04-1.17)$ & $<0.001$ & $1.01(0.89-1.15)$ & $9.00 \mathrm{E}-01$ & $1.13(1.06-1.20)$ & $<0.001$ \\
\hline $70-79$ & $1.16(1.09-1.23)$ & $<0.001$ & $1.04(0.91-1.19)$ & $5.70 \mathrm{E}-01$ & $1.19(1.12-1.26)$ & $<0.001$ \\
\hline$>79$ & $1.17(1.10-1.24)$ & $<0.001$ & $1.00(0.87-1.17)$ & $9.60 \mathrm{E}-01$ & $1.19(1.12-1.27)$ & $<0.001$ \\
\hline
\end{tabular}

2 We included all variables in the SH multivariate analysis. HR, Hazard ratio; 95\%Cl, 95\%confidence index; Significant results ( $p<0.05$ ) were bolded. 


\section{Table 3(on next page)}

Multivariate SH analyses of CSS for each variable in VEOPC patients

We included all variables in the SH multivariate analysis. HR, Hazard ratio; $95 \% \mathrm{Cl}$, $95 \%$ confidence index; Significant results $(p<0.05)$ were bolded. 
1 Table 3. Multivariate SH analyses of CSS for each variable in VEOPC patients

\begin{tabular}{llr}
\hline Characteristics & HR $(95 \% \mathrm{Cl})$ & p value \\
\hline Sex & Reference & \\
Male & $\mathbf{0 . 8 7}(\mathbf{0 . 7 7 - 0 . 9 8 )}$ & $\mathbf{0 . 0 2 1}$ \\
Female & & \\
Race & Reference & \\
Caucasian & $1.02(0.84-1.23)$ & 0.880 \\
African American & $1.00(0.67-1.50)$ & 1.000 \\
American Indian/Alaska Native & $\mathbf{1 . 5 4}(\mathbf{1 . 3 0 - 1 . 8 3})$ & $<\mathbf{0 0 1}$ \\
Asian or Pacific Islander & & \\
Location & Reference & \\
Head of pancreas & $0.89(0.73-1.08)$ & 0.220 \\
Body of pancreas & $1.07(0.87-1.32)$ & 0.530 \\
Tail of pancreas & $\mathbf{2 . 0 1}(\mathbf{1 . 3 4 - 3 . 0 2})$ & $\mathbf{0 . 0 0 1}$ \\
Pancreatic duct & $0.65(0.33-1.29)$ & 0.220 \\
Other specified parts of pancreas & $0.92(0.72-1.18)$ & 0.520 \\
Overlapping lesion of pancreas & $\mathbf{1 . 2 8}(\mathbf{1 . 0 3 - 1 . 6 0 )}$ & $\mathbf{0 . 0 2 5}$ \\
Pancreas, NOS &
\end{tabular}

\section{Surgery}

No surgery

Reference

Local or partial pancreatectomy

$0.40(0.26-0.61)$

$<0.001$

Local or partial pancreatectomy and duodenectomy

$0.46(0.36-0.60)$

$<0.001$

Total pancreatectomy with or without gastrectomy or

$0.41(0.28-0.61)$

$<0.001$

duodenectomy

$0.77(0.36-1.64)$

0.490

\section{Tumor size}

$<=2 \mathrm{~cm}$

Reference

2 to 4

1.07 (0.87-1.32)

0.510

4 to 6

$1.11(0.88-1.41)$

0.360

$>6$

1.28 (0.94-1.74)

0.110

Unknown

$1.01(0.78-1.32)$

0.930

\section{LNR}

0

Reference

$<=0.2$

1.39 (1.02-1.90)

0.037

$0.2-0.4$

1.80 (1.30-2.51)

$<0.001$

0.4-1

1.90 (1.37-2.64)

$<0.001$

No nodes were examined

1.33 (1.04-1.70)

0.021

\section{T stage}

T1-T2

Reference

T3-T4

$1.16(0.98-1.37)$

0.082

Unknown

$0.95(0.73-1.22)$

0.680 


\begin{tabular}{|c|c|c|}
\hline \multicolumn{3}{|l|}{ N stage } \\
\hline NO & Reference & \\
\hline $\mathrm{N} 1$ & $1.12(0.95-1.31)$ & 0.170 \\
\hline Unknown & $1.34(1.09-1.66)$ & 0.005 \\
\hline \multicolumn{3}{|l|}{ M stage } \\
\hline M0 & Reference & \\
\hline M1 & $1.48(1.25-1.75)$ & $<0.001$ \\
\hline Unknown & $1.09(0.60-1.96)$ & 0.780 \\
\hline \multicolumn{3}{|l|}{ Grade } \\
\hline$|-I|$ & Reference & \\
\hline III-IV & $1.29(1.07-1.56)$ & 0.007 \\
\hline Unknown & $1.09(0.93-1.29)$ & 0.290 \\
\hline \multicolumn{3}{|c|}{ Chemotherapy } \\
\hline No & Reference & \\
\hline Yes & $0.63(0.53-0.76)$ & $<0.001$ \\
\hline \multicolumn{3}{|c|}{ Radiotherapy } \\
\hline No & Reference & \\
\hline Yes & $1.09(0.94-1.25)$ & 0.250 \\
\hline \multicolumn{3}{|c|}{ Marital status } \\
\hline Married & Reference & \\
\hline Single & $1.15(1.02-1.30)$ & 0.021 \\
\hline
\end{tabular}

2 We included all variables in the $\mathrm{SH}$ multivariate analysis. $\mathrm{HR}$, Hazard ratio; $95 \% \mathrm{Cl}, 95 \%$ confidence index;

3 Significant results $(p<0.05)$ were bolded. 
Figure 1

CIF plots of the association between age and the prognosis of PDAC patients

$\mathrm{a}-\mathrm{c}$, The CIF plots of the association between age and the prognosis of all cohort (a), patients with surgery (b) or without surgery (c). 


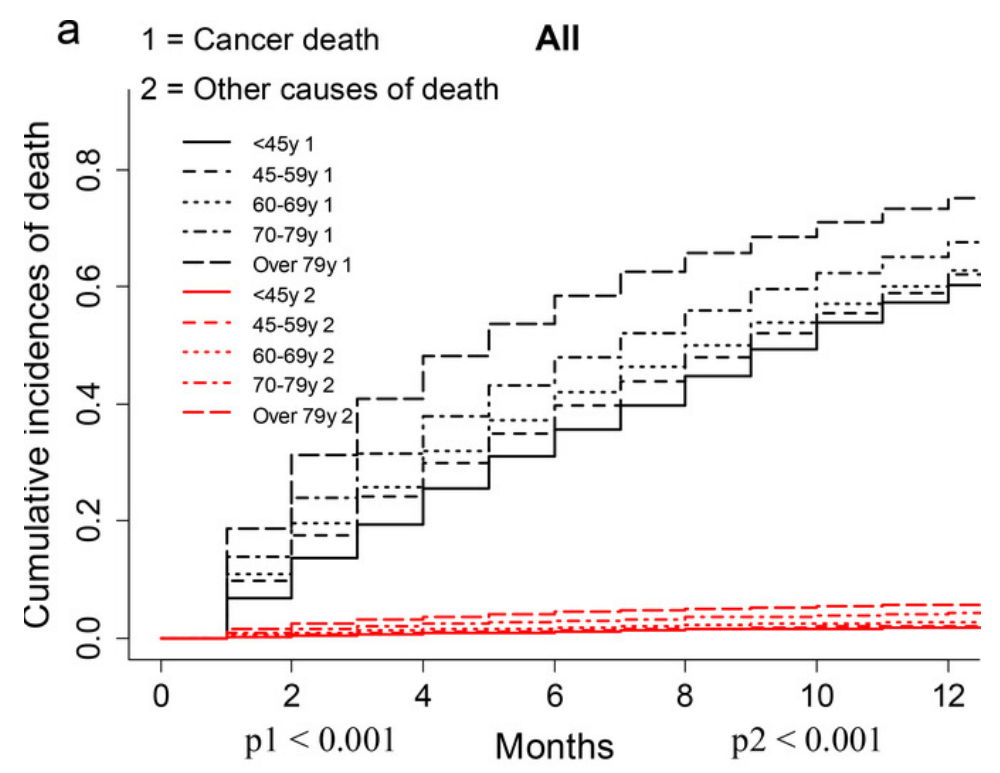

\section{b 1 = Cancer death With Surgery}
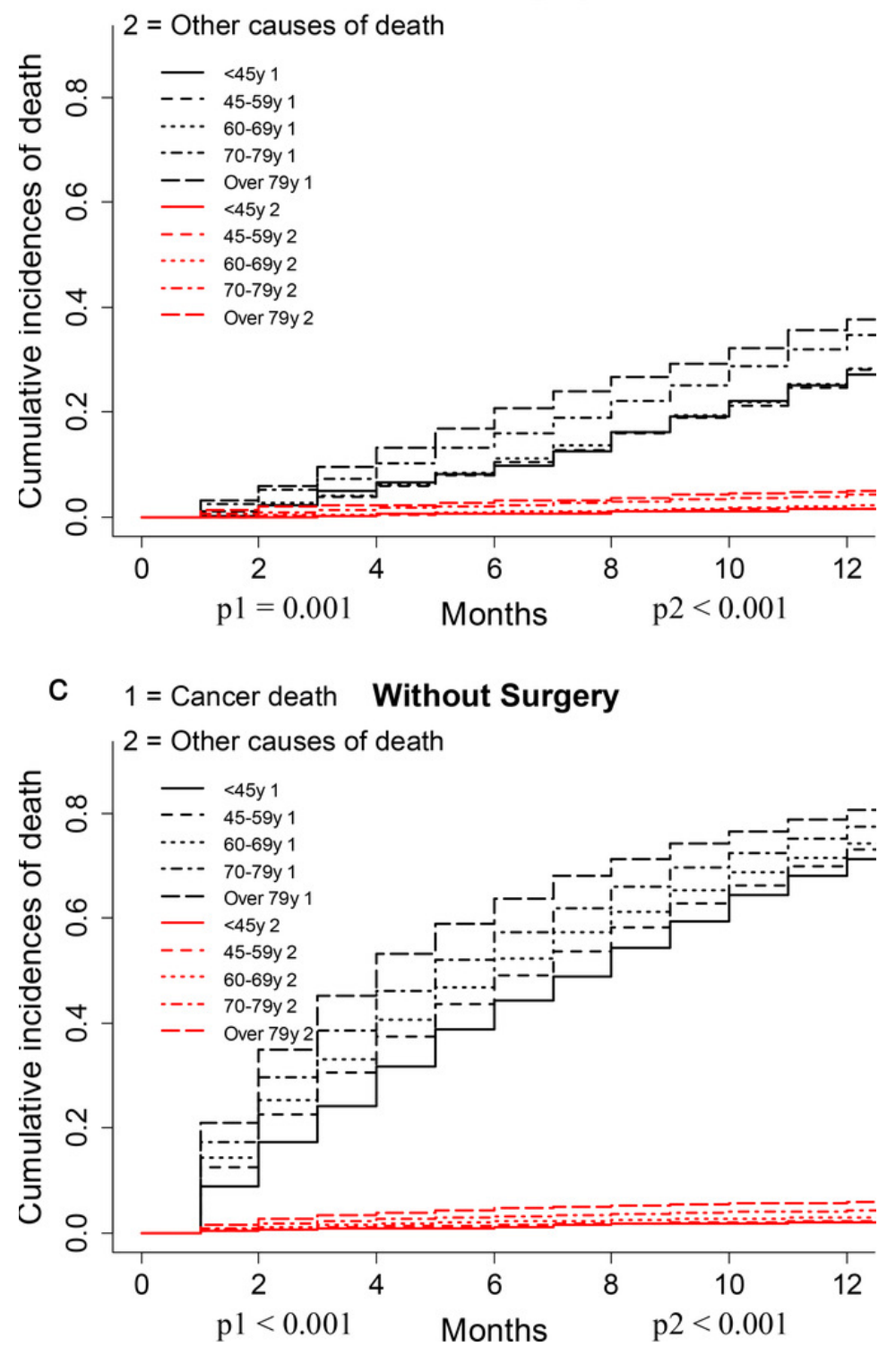

Peer) reviewing PDF | (2019:06:38653:1:1:REVIEW 5 Dec 2019) 


\section{Figure 2}

Nomogram for predicting 6 months, 12 months, 18 months and 24 months cancer specific death of VEOPC patients

The nomogram is used by summing the points identified on the top scale for each independent variable and drawing a vertical line from the total points scale to the 6 months, 12 months, 18 months and 24 months cancer specific death to obtain the probability of survival. The total points projected to the bottom scale indicate the probability of the 6 months, 12 months, 18 months and 24 months cancer specific death. Sex: $1=$ Male, $2=$ Female; Race: 1 = Caucasian, 2 = African American, $N=$ Other race; Location: $250=$ Head of pancreas, $251=$ Body of pancreas, $252=$ Tail of pancreas, $253=$ Pancreatic duct, $257=$ Other specified parts of pancreas, $258=$ Overlapping lesion of pancreas, $259=$ Pancreas, NOS; Surgery, $0=$ No surgery, $1=$ Local or partial pancreatectomy, $2=$ Local or partial pancreatectomy and duodenectomy, $3=$ Total pancreatectomy with or without gastrectomy or duodenectomy, $4=$ Pancreatectomy NOS or surgery NOS; Tumor size: $2=<=2 \mathrm{~cm}, 4=$ $2 \mathrm{~cm}$ to $4 \mathrm{~cm}, 6=4 \mathrm{~cm}$ to $6 \mathrm{~cm}, 8=>6 \mathrm{~cm}, 9=$ Unknown; LNR: $0=0,1=<=0.2,2=0.2-0.4$, $3=>0.4,4=$ No nodes were examined; $\mathrm{T}$ stage: $1=\mathrm{T} 1-\mathrm{T} 2,2=\mathrm{T} 3-\mathrm{T} 4,9=$ Unknown; $\mathrm{N}$ stage: $0=\mathrm{NO}, 1=\mathrm{N} 1,9=$ Unknown; $\mathrm{M}$ stage: $0=\mathrm{M} 0,1=\mathrm{M} 1,9=$ Unknown; Grade: $1=\mathrm{I}-\mathrm{II}$, $2=I I I-I V, 9=$ Unknown; Chemotherapy: $0=$ none/unknown and $1=$ yes; Radiotherapy: $0=$ none/unknown or refused, 1 = beam radiation or combination of beam with implants or isotopes or radiation with method or source not specified or radioactive implants or radioisotopes and $\mathrm{N}=$ Recommended, unknown if administered; Marital status: $0=$ married; $1=$ widowed or single (never married or having a domestic partner) or divorced or separated. The asterisk means significant. 


\section{SH model}

Sex*

$\operatorname{Race}^{\star * *}$

Location** $^{\star *}$

Surgery ${ }^{* * *}$

Tumor_size*

$\mathrm{LNR}^{\star \star *}$

T_stage*

N_stage ${ }^{* * *}$

M_stage ${ }^{* \star *}$

Grade** $^{* *}$

Chemotherapy $y^{* * *}$

Radiotherapy

Marital_status**

\section{Points}

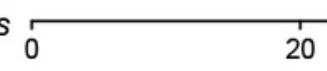

40

\section{0}

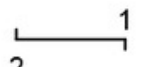

2

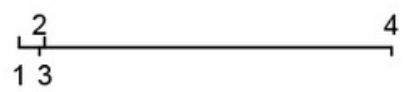

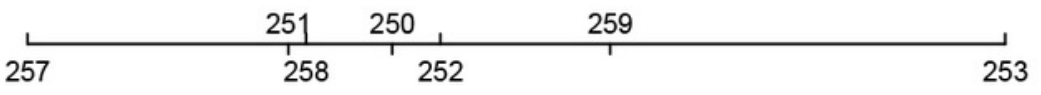
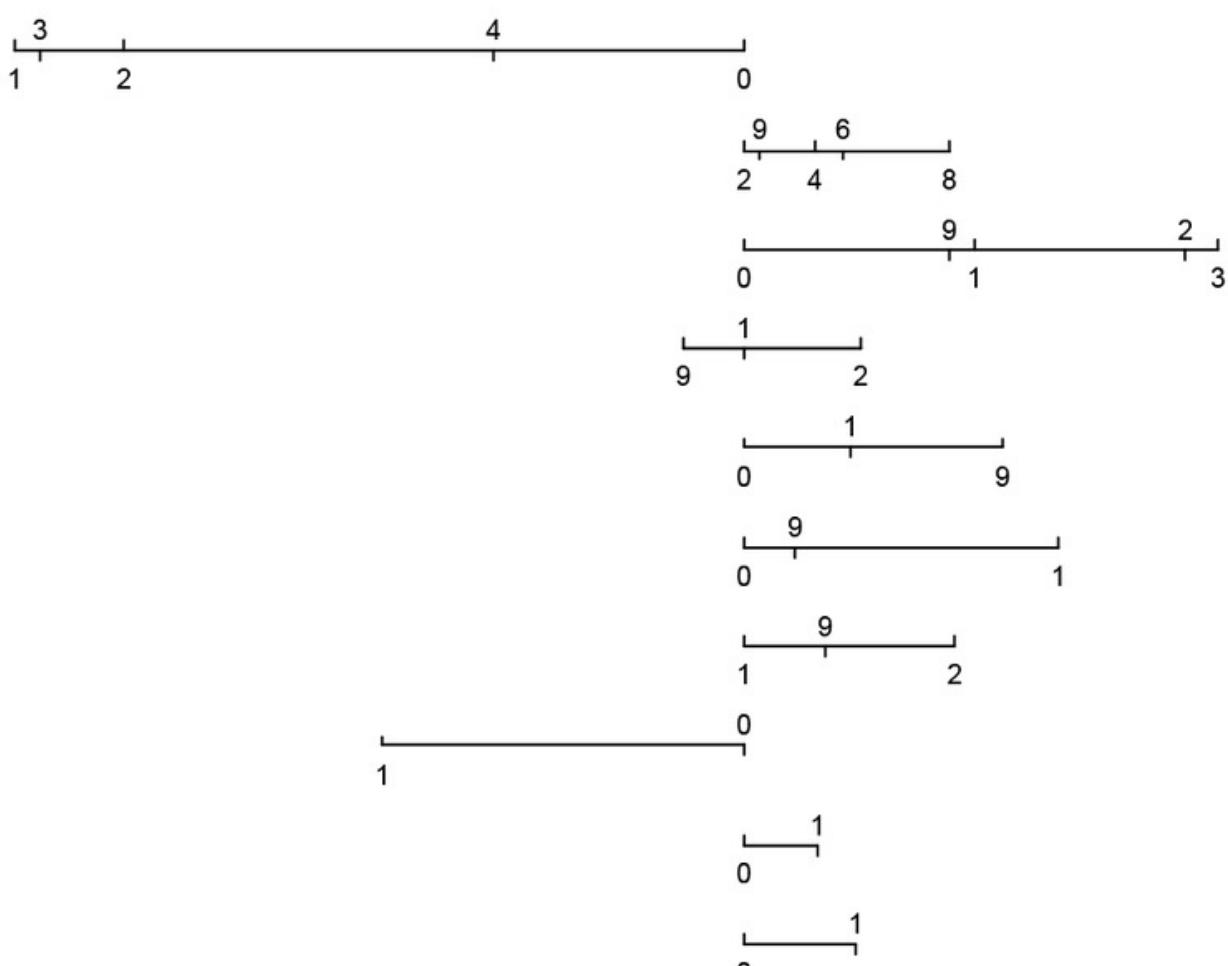

0

Total-points-to-outcome nomogram:

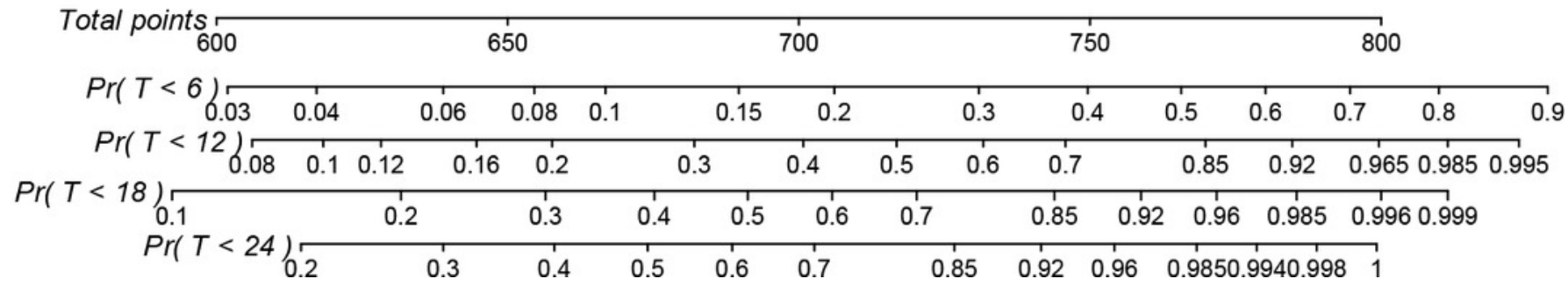


Figure 3

Calibration curves and AUC plots for internal validation of $\mathrm{SH}$ based nomograms

a-d, the calibration plots for 6 months (a), 12 months (b) , 18 months (c) and 24 months (d) cancer specific death prediction of VEOPC patients; The x-axis shows the nomogram predicted probability while the y-axis is the actual survival estimated by the SH method, the black thick line overlaps the grey line indicating near perfect calibration; e, time dependent AUC plots of SH-based nomograms.

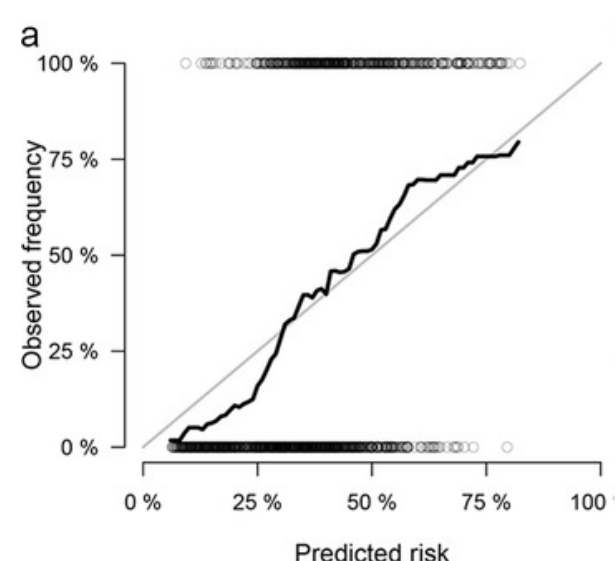

Predicted risk

d

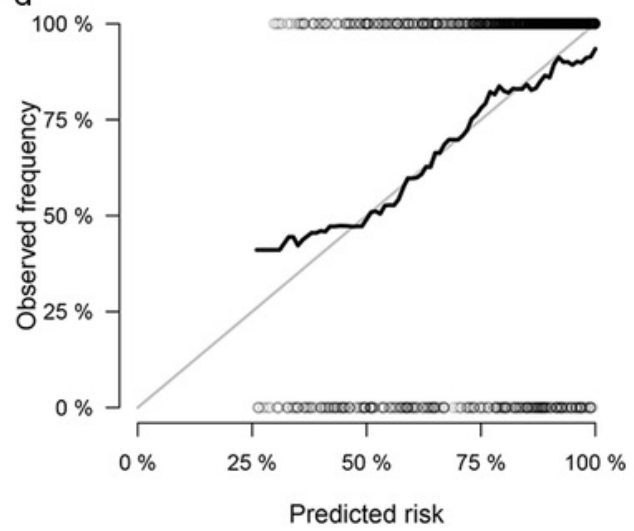

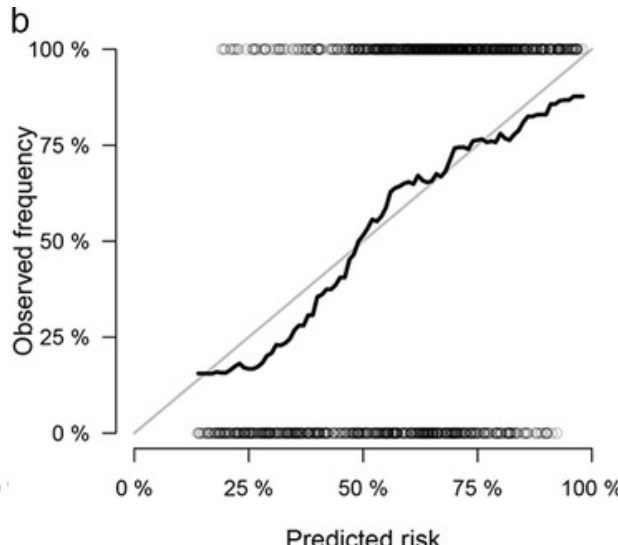

e

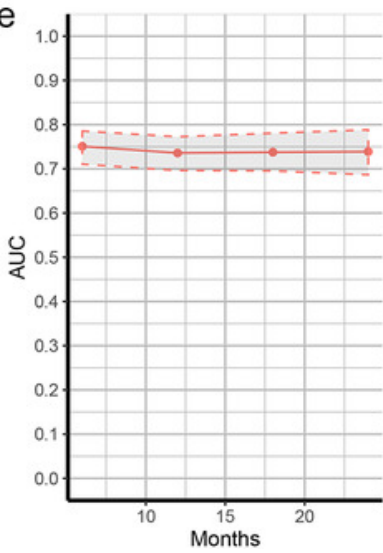

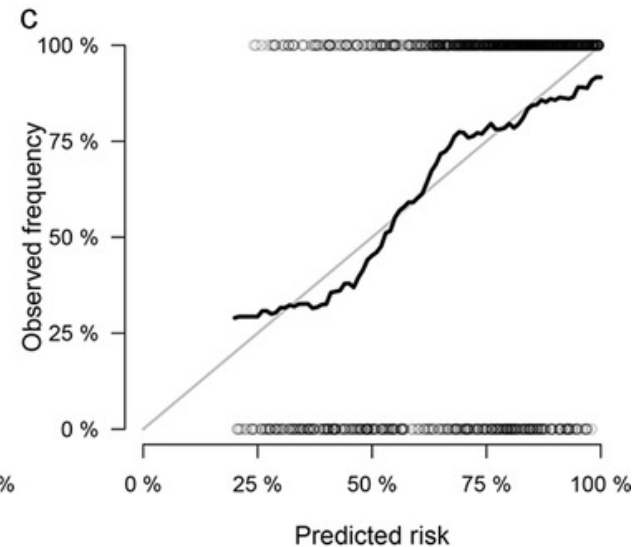

model

Fine-Gray 
Figure 4

CIF plots based on the competing nomogram scores for the VEOPC patients

the whole cohort of VEOPC patients were divided into three equally distributed groups. The groups were named "High", "Intermediate" and "Low" according to the levels of patients' competing nomogram scores.

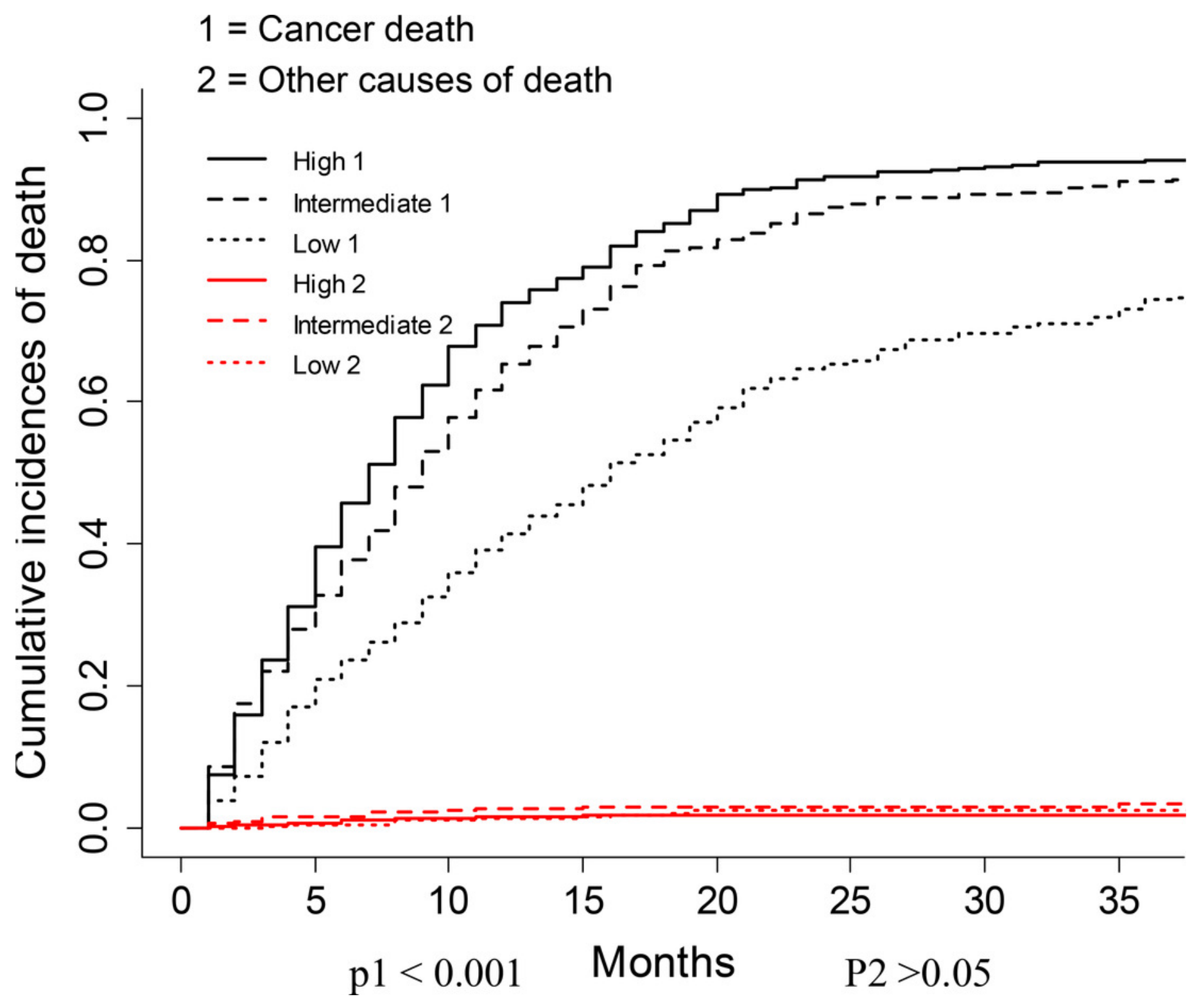

